\title{
Neural Stem and Progenitor Cells with High Proliferation Potential in Injured Areas in a Model of Neonatal Hypoxic-Ischemic Encephalopathy
} Jun Kido ${ }^{1 *}$ and Tomoaki Matsumoto

${ }^{1}$ Department of Pediatrics, Graduate School of Medical Sciences, Kumamoto University, Kumamoto City, Kumamoto, Japan ${ }^{2}$ Pediatric Division, Aso Spa Hospital, Aso City, Kumamoto, Japan

\begin{abstract}
Objective: Neonatal hypoxic-ischemic encephalopathy causes death and severe neurological sequelae including cerebral palsy, epilepsy, and mental retardation. Because hypothermia therapy is the only effective treatment for neonatal hypoxic-ischemic encephalopathy and its efficacy is limited in severe cases, a more effective treatment is needed. Neural stem and progenitor cells are important for neuroprotection and neuronal replacement following hypoxic-ischemic encephalopathy. The aim of this study was to confirm the presence of neural stem cells and the acceleration of their mitotic activity in injured areas in a model of neonatal hypoxic-ischemic encephalopathy.

Method: Hypoxic-ischemic encephalopathy was induced in rat pups by left common carotid artery occlusion followed by hypoxia. Following ischemic injury, brains were sectioned, and columns of tissue were excised from infarct areas for cell culture using the neurosphere assay. Cell clusters were analyzed by immunocytochemistry and reverse transcription polymerase chain reaction. Clustered cells were also tested for their ability to differentiate into neurons, astrocytes, and oligodendrocytes.

Results: Neurosphere-like cell clusters from infarct areas of ischemia-injured animals were dramatically increased compared to samples from sham-operated control animals. Clusters included cells immunopositive for bromodeoxyuridine, nestin, neural/glial antigen 2, $\beta$-III tubulin, glial fibrillary acidic protein, oligodendrocyte marker O4, vimentin, and ionizing calcium-binding adaptor molecule 1. Cell clusters also expressed mRNA for NANOG, (sex determining region $Y$ )-box 2 , octamer-binding transcription factor $3 / 4$, and Rex 1 , similar to embryonic stem cells, and could be differentiated into neurons, astrocytes or oligodendrocytes that expressed mRNAs for nestin, $\beta$-III tubulin, glial fibrillary acidic protein, and myelin basic protein.

Conclusion: We demonstrate the presence of many neural stem and progenitor cells with enhanced proliferative potential in infarct brain areas of a rat model of neonatal hypoxic-ischemic encephalopathy. Activation of neural stem and progenitor cells in the infarct brain area may contribute to neuroprotection and regeneration.
\end{abstract}

Keywords: Hypoxic-ischemic encephalopathy; Levine procedure; Nestin; Neural stem cell; Proliferation potential; Neurosphere; Neural differentiation

\section{Introduction}

Neonatal hypoxic-ischemic encephalopathy (HIE) is the most common neonatal encephalopathy and can cause death and severe neurological sequelae such as cerebral palsy, epilepsy, and mental retardation. The frequency of neonatal encephalopathy in developed countries is estimated at 3 per 1000 live births and the frequency of HIE is 1.5 per 1000 live births [1]. At present, hypothermia therapy is the only effective treatment for HIE [2-4] and is commonly used worldwide. However, the efficacy of hypothermia therapy is limited in severe cases; thus, a more effective treatment based on inherent brain protective mechanisms is needed to supplement hypothermia therapy.

Recent progress in neural stem cell research suggests the potential use of neural regeneration treatment for hypoxic-ischemic brain injury. Continuous neurogenesis occurs in the subventricular zone (SVZ) of the lateral ventricle and the subgranular zone of the hippocampal dentate gyrus in the adult mammalian brain [5-7]. Neurogenesis can also be activated by stimuli such as hypoxia and ischemic injury [8] Neurogenesis after ischemic brain injury occurs in three steps: (1) proliferation of neural stem and progenitor cells in the SVZ $[9,10],(2)$ migration of neural stem and progenitor cells to the injured area $[11,12]$, and (3) differentiation into mature neurons in the injured area $[13,14]$. In the adult brain, neurons differentiated from stem and progenitor cells integrate into the neural network at the ischemia-injured area and participate in the recovery of neurological function $[10,15]$. In HIE models, however, increased proliferation of neural stem and progenitor cells in the SVZ and progenitor cell migration to injured areas are observed, but neuronal differentiation and survival are limited [16,17]. The poor viability of newborn neurons in HIE models may be caused by an unfavorable microenvironment and lack of appropriate trophic support. As support for this notion, erythropoietin treatment enhances revascularization to ischemia-injured areas after HIE induction and thereby promotes neuronal replacement $[18,19]$. However, the reason for the inability of neural progenitor cells to survive and differentiate in injured areas in HIE models is not fully understood. Because neural stem cells cannot be directly detected because of the lack of a specific marker, it is not clear how many stem cells migrate to injured areas after HIE induction.

Different organs throughout the body contain tissue-specific stem

*Corresponding author: Jun Kido, Department of Pediatrics, Graduate School of Medical Sciences, Kumamoto University, 1-1-1 Honjo, Kumamoto City, Kumamoto Prefecture 860-8556, Japan, Tel: +81-096-373-5191; Fax: +81-096-373-5336; E-mail: kidojun@fc.kuh.kumamoto-u.ac.jp

Received November 27, 2015; Accepted January 11, 2016 ; Published January 18, 2016

Citation: Kido J, Matsumoto T (2016) Neural Stem and Progenitor Cells with High Proliferation Potential in Injured Areas in a Model of Neonatal Hypoxic-Ischemic Encephalopathy. J Neurol Neurophysiol 7: 348. doi:10.4172/2155-9562.1000348

Copyright: (c 2016 Kido J, et al. This is an open-access article distributed under the terms of the Creative Commons Attribution License, which permits unrestricted use, distribution, and reproduction in any medium, provided the original author and source are credited. 
Citation: Kido J, Matsumoto T (2016) Neural Stem and Progenitor Cells with High Proliferation Potential in Injured Areas in a Model of Neonatal Hypoxic-Ischemic Encephalopathy. J Neurol Neurophysiol 7: 348. doi:10.4172/2155-9562.1000348

cells whose mitotic activity is stimulated by injury to promote tissue repair [18-24]. In adult stroke models, cells derived from ischemiainjured brain tissue form neurosphere-like clusters in neurosphere assays; these cells can be differentiated into neurons, astrocytes, and oligodendrocytes [25]. Because ischemia-injured areas in HIE models contain many neural progenitor cells [12], we hypothesized that we could isolate neural stem cells derived from ischemia-injured areas. The aim of this study was to confirm the presence of neural stem cells in injured areas after HIE induction and examine their mitotic activity and differentiation potential. Neural stem and progenitor cells are thought to migrate to injured brain areas to not only to compensate for neuronal loss but also to protect surviving neurons. The presence of neural stem cells with increased mitotic activity in ischemia-injured areas in HIE models would suggest that progenitor cells may be used for neuroprotection or neuronal replacement in the treatment of HIE.

\section{Materials and Method}

This article is partly adhered to current RIGOR guidelines [26,27]. 1) Blinding of the study: no blinded conduct of the experiment and no blinded assessment of koutcome. 2) Randomization: Animals should be assigned randomly to the Sham-operated control and HIE groups. 3) Power analysis and 4) Statistical analysis are performed in the Shamoperated control and HIE groups.

\section{Animal model}

Pregnant Wistar rats were purchased from Japan Charles River Co., Ltd. (Tsukuba, Japan). On postnatal day 7, each pup was subjected to a modified Levine procedure to induce HIE $[28,29]$. Briefly, after induction of anesthesia with isoflurane, the left common carotid artery of each pup was sectioned between double ligatures with 5-0 surgical silk. The pups were allowed to recover for $1 \mathrm{~h}$ and were then exposed to an additional $2 \mathrm{~h}$ of hypoxia ( $8 \%$ oxygen, $92 \%$ nitrogen) in a plastic container at $37 \mathrm{C}$. Sham-operated control animals were treated identically, but did not undergo sectioning of the left common carotid artery or subsequent hypoxia. The experimental protocol was approved by the ethics committee for animal research at Kumamoto University

\section{Confirmation of ischemic tissue damage}

Three days after HIE induction, the animals' forebrains were removed under deep anesthesia with diethyl ether. Coronal sections (2$\mathrm{mm}$ thick) were obtained $-3 \mathrm{~mm}$ from the bregma and stained with $1 \%$ triphenyltetrazolium chloride (TTC; Wako Pure Chemical Industries, Ltd., Osaka, Japan) to identify the ischemia-injured area [30]. Brain images were obtained using a stereoscopic microscope (SZ61-TRC-CK, Olympus, Tokyo, Japan).

\section{Primary cell culture and determination of cell cluster number}

Three days after HIE induction, 6 columnar tissue samples (1.4 $\mathrm{mm}$ in diameter) between -3 and $-1 \mathrm{~mm}$ from the bregma were excised from the SVZ, cerebral cortex, and striatum of both the ischemic and non-ischemic hemispheres (Figure 1A, areas a-f). Each sample was passed through a 29-gauge needle to dissociate the cells. The resulting cell suspensions were incubated according to previously described neurosphere culture assay protocols [31-33]. Briefly, cell suspensions were placed in uncoated 6-well plates and incubated for 14 days at $37^{\circ} \mathrm{C}$ with $5 \% \mathrm{CO}_{2}$ with Neurobasal Medium (Thermo Fisher Scientific, Inc., Waltham, MA, USA) containing epidermal growth factor (EGF; 20 ng/mL; Sigma-Aldrich, St. Louis, MO, USA), basic fibroblast growth factor (bFGF; $20 \mathrm{ng} / \mathrm{mL}$; R\&D Systems, Inc., Minneapolis, MN, USA), N2 supplement (1\%), B27 supplement (2\%), Insulin-TransferrinSelenium-X supplement (1\%), and Glutamine-Penicillin-Streptomycin (1\%; all from Thermo Fisher Scientific). To determine the peak of cell proliferation potential, total numbers of cell clusters exceeding 50 冈m in diameter were counted for 9 days using a phase contrast microscope (IX70, Olympus, Tokyo, Japan). In order to investigate the biological properties of the primary cell clusters derived from the ischemia-

A

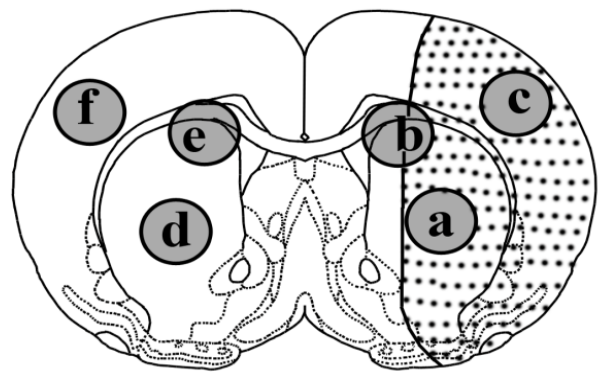

Anterior
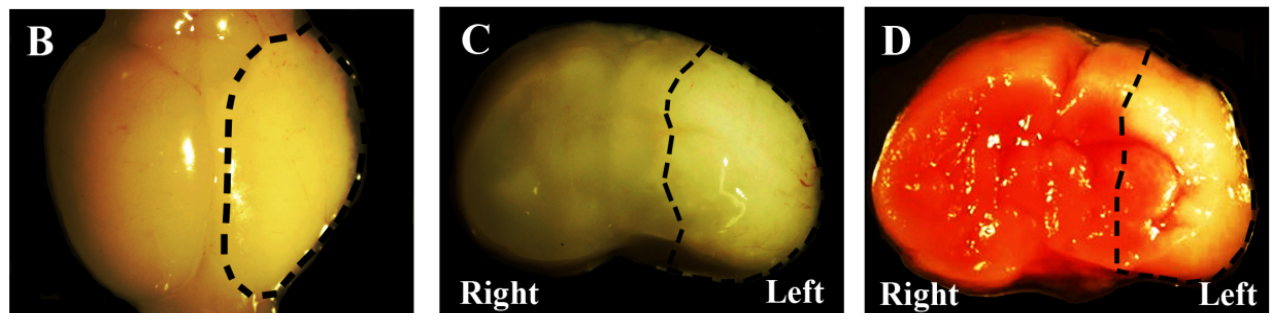

Figure 1: Rat model of neonatal hypoxic-ischemic encephalopathy (HIE) (A) The dotted area represents the infarct region. Areas a and c, but not b, are infarct regions (B-D) Stereomicroscopic images of the brain of a Wistar rat 3 days after HIE induction. Dashed lines indicate the infarct area. (B) Whole brain; (C) coronal section; (D) triphenyltetrazolium chloride (TTC)-stained coronal section; infarct area is not stained. 
injured areas in the HIE model, differentiation was induced by omitting EGF and bFGF in a laminin-coated dish for 7 days and confirmed by immunocytochemistry as described previously [34].

\section{Secondary cell culture}

To investigate the biological properties of cell clusters derived from ischemia-injured areas in the HIE model, secondary cell culture using the neurosphere culture protocol was performed as described previously [32]. Cell clusters derived from ischemia-injured areas of the cortex were collected at the peak of cell proliferation potential, and single-cell suspensions were prepared by isolating 1 or 2 cells in $10 \mu$ volumes. These single-cell suspensions were placed in 96-well plates with $100 \mu \mathrm{l}$ of medium containing EGF and b-FGF, as previously described. After $8 \mathrm{~h}$, wells containing single viable cells were marked, and the number of wells containing cell clusters was recorded for 30 days. On alternate days, half of the growth medium was exchanged for fresh medium. A differentiation study was also performed on these secondary cultures by omitting EGF and bFGF.

\section{MTT cell proliferation assay}

The yellow tetrazolium MTT (3-[4,5-dimethylthiazol-2-yl]2,5-diphenyltetrazolium bromide; Wako Pure Chemical Industries) is reduced by metabolically active cells, in part by mitochondrial dehydrogenase enzymes, to generate reducing equivalents such as $\mathrm{NADH}$ and NAPDH. The resulting intracellular purple formazan can be solubilized and quantified using a spectrophotometer. The MTT cell proliferation assay measures the cell proliferation rate. The linear relationship between cell number and signal produced has been established, thus allowing accurate quantification of cell proliferation rate [35].

Cell suspensions were prepared from SVZ samples isolated from sham-operated controls and the ischemic and non-ischemic hemispheres of HIE model animals 3 days after HIE induction. Each suspension was prepared at a density of 10,000 cells $/ 100 \mu \mathrm{L}$, plated in 96-well plates, and cultured using the above neurosphere culture protocol. On cell culture day $4,10 \mu \mathrm{L}$ of MTT solution $(2.5 \mathrm{mg} / \mathrm{mL})$ was added to each well, and the plates were incubated at $37^{\circ} \mathrm{C}$ for $3 \mathrm{~h}$ to allow mitochondrial uptake of MTT. An equal volume $(100 \mu \mathrm{L})$ of acidified isopropanol was then added to each well in order to induce cytolysis, and the absorbance at $570 \mathrm{~nm}$ was measured for each well.

\section{Immunocytochemistry}

Cell clusters were attached to glass slides using cytospin preparations. After fixation with $4 \%$ paraformaldehyde and blocking with $5 \%$ bovine serum albumin (BSA) in phosphate-buffered saline (PBS) for 1 hour, sections were incubated with primary antibodies at $4^{\circ} \mathrm{C}$ overnight followed by the appropriate secondary antibodies for 1 hour at room temperature. Primary antibodies included the following: chicken antinestin (1:2000; Aves Labs, Inc., Chicago, IL, USA), mouse anti- $\beta$-IIItubulin (1:100; Merck Millipore, Inc., Darmstadt, Germany), chicken anti-microtubule-associated protein 2 (MAP2; 1:1000; Aves Labs), rabbit anti-glial fibrillary acidic protein (GFAP; 1:100; Epitomics, Inc., Burlingame, CA, USA), mouse anti-neural/glial antigen 2 (NG2; 1:100; Abcam, Inc., Cambridge, MA, USA), mouse anti-oligodendrocyte marker O4 (O4; 1:100; Merck Millipore), chicken anti-vimentin (1:1000; Aves Labs), and rabbit anti-ionizing calcium-binding adaptor molecule 1 (Iba1; 1:500; Wako Pure Chemical Industries). Secondary antibodies used were Alexa Fluor 488 or 594 anti-mouse, rabbit, or chicken (all 1:2000; Thermo Fisher Scientific). Nuclei were stained with 4',6-diamidino-2-phenylindole (DAPI; Thermo Fisher Scientific).
To identify newborn cells in the cell clusters, dissociated cells derived from the ischemia-injured area of the cortex were incubated for $96 \mathrm{~h}$ with 5-bromo-2'-deoxyuridine (BrdU; $10 \mu \mathrm{M}$; Nacalai Tesque, Inc., Kyoto, Japan). After attachment and fixation of the cell clusters as described above, the clusters were denatured with $2 \mathrm{~N} \mathrm{HCl}$ for 30 minutes at $37^{\circ} \mathrm{C}$ and incubated with 5\% BSA in PBS for 1 hour followed by mouse anti-BrdU antibody (1:100; Merck Millipore) at $4^{\circ} \mathrm{C}$ overnight. The cells were then incubated with Alexa Fluor 488 anti-mouse and visualized using a laser scanning confocal microscope (FLUOVIEW FV500, Olympus, Tokyo, Japan).

\section{Reverse transcriptase-polymerase chain reaction (RT-PCR)}

Total RNA was extracted from cell clusters using an RNeasy Mini Kit (QIAGEN Inc., Valencia, CA, USA). cDNA was synthesized from the purified RNA using the ThermoScript RT-PCR System (Thermo Fisher Scientific). cDNA was amplified under the following conditions: denaturation for $40 \mathrm{~s}$ at $94^{\circ} \mathrm{C}$; annealing for $40 \mathrm{~s}$ at $52-60^{\circ} \mathrm{C}$ (primerspecific); and extension for $1 \mathrm{~min}$ at $72^{\circ} \mathrm{C}$ (35 cycles) [36-41]. Primer sequences were as follows:

NANOG forward, TCTCCTCCGCCTTCCTCT and NANOG reverse, TTGCCTCTGAAACCTATCCTTG (amplicon size: 204 bp; annealing temperature: $53^{\circ} \mathrm{C}$ ); (sex determining region $\mathrm{Y}$ )-box 2 (Sox2) forward, GGGCTCTGTGGTCAAGTC and Sox2 reverse, TAGTCGGCATCACGGTTT (amplicon size: $435 \mathrm{bp}$; annealing temperature: $\left.60^{\circ} \mathrm{C}\right)$; octamer-binding transcription factor $3 / 4($ Oct $3 / 4)$ forward, AGGCAGGAGCACGAGTGGA and Oct3/4 reverse, CGAAGCGGCAGATGGTTGT (amplicon size: 264 bp; annealing temperature: $59^{\circ} \mathrm{C}$ ); Rex1 forward, AAATCATGACGAGGCAAGGC and Rex1 reverse, TGAGTTCGCTCCAACAGTCT (amplicon size: $350 \mathrm{bp}$; annealing temperature: $52^{\circ} \mathrm{C}$ ); Kruppel-like factor 4 (KLF4) forward, CAGACCTGGAAAGTGGTGG and KLF4 reverse, ACCTGTGTTGCCCGCAGCC (amplicon size: 283 bp; annealing temperature: $52^{\circ} \mathrm{C}$ ); glyceraldehyde-3-phosphate dehydrogenase (GAPDH) forward, CCTCTGGAAAGCTGTGGCGT and GAPDH reverse, TTGGAGGCCATGTAGGCCAT (amplicon size: $430 \mathrm{bp}$; annealing temperature: 56 $6^{\circ}$ ); nestin forward, CAGGCTTCTCTTGGCTTTCTGG and nestin reverse, TGGTGAGGGTTGAGGTTTGT (amplicon size: $431 \mathrm{bp}$; annealing temperature: $\left.56^{\circ} \mathrm{C}\right) ; \quad \beta$-III-tubulin forward, TGCGTGTGTACAGGTGAATGC and $\beta$-III-tubulin reverse, AGGCTGCATAGTCATTTCCAAG (amplicon size: $240 \mathrm{bp}$; annealing temperature: $55^{\circ} \mathrm{C}$ ); GFAP forward, ACCTCGGCACCCTGAGGCAG and GFAP reverse, CCAGCGACTCAACCTTCCTC (amplicon size: $141 \mathrm{bp}$; annealing temperature: $59^{\circ} \mathrm{C}$ ); myelin basic protein (MBP) forward, CAAGGTACCCTGGCTAAAG and MBP reverse, CCCAGCTTAAAGATTTTGG (amplicon size: $372 \mathrm{bp}$; annealing temperature: $55^{\circ} \mathrm{C}$ ); $\beta$-actin forward, CTAAGGCCAACCGTGAAAAGATGAC and $\beta$-actin reverse, TGGGTACATGGTGGTGCCACCAGAC (amplicon size: 587 bp; annealing temperature: $57^{\circ} \mathrm{C}$ ).

\section{Statistical analysis}

Data were analyzed using the Shapiro-Wilk normality test. Normalized results are presented as the mean \pm standard error (SE) in Figure 2. Statistical comparisons among groups (HIE ipsilateral and contralateral hemispheres and sham-operated animals) were performed using the Games-Howell test or Mann-Whitney U test in SPSS version 17.0 (IBM, Chicago, IL, USA). $P$ values $<0.05$ were considered statistically significant. 
Day 2
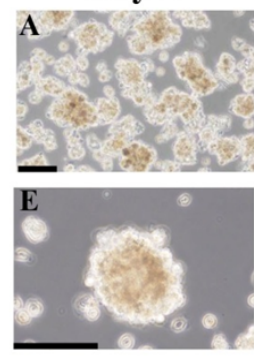

I.SVZ

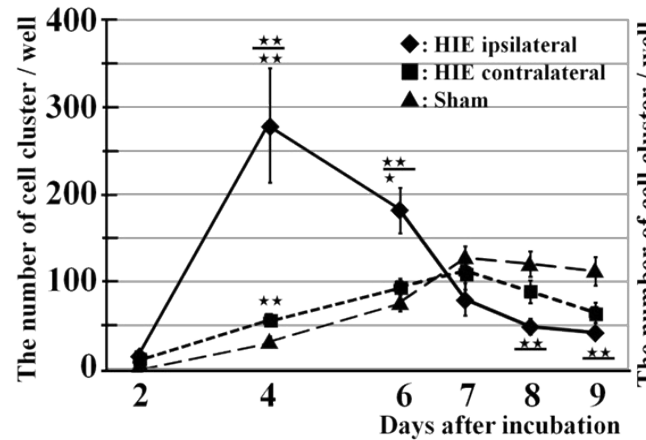

K.Cortex

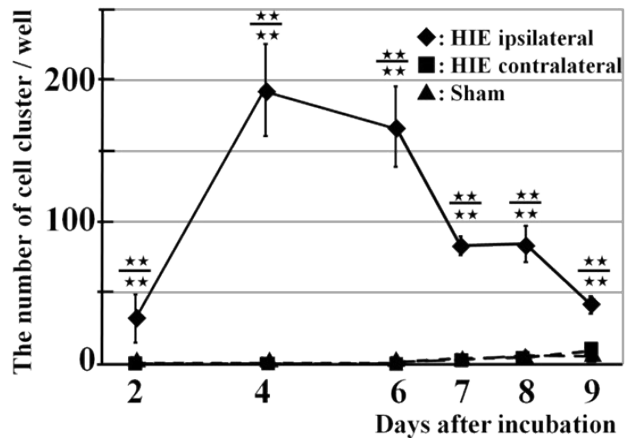

BrdU

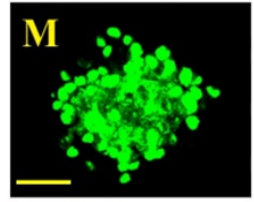

Day 4
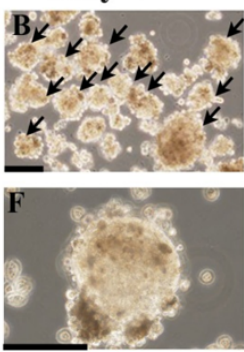

J.Striatum

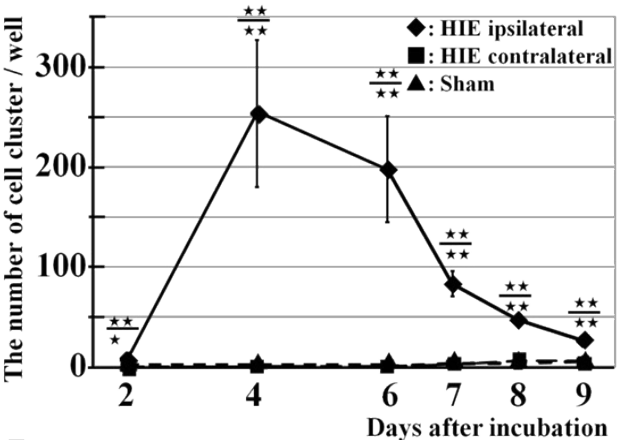

L.

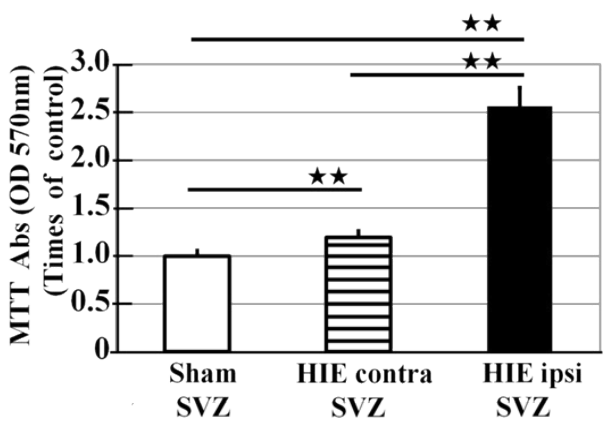

\section{Day14}

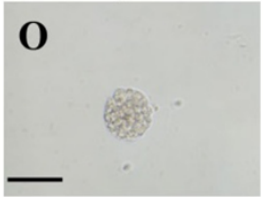

Day 14

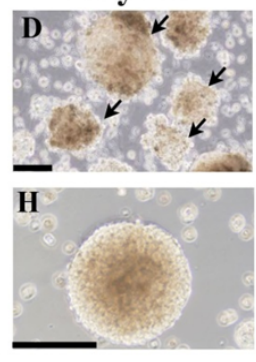

Figure 2: Formation of neurosphere-like cell clusters by cells harvested from HIE model animals. (A-H) Cells obtained from the infarct cortex form cell clusters in neurosphere culture assays that increase in diameter over time. Images were obtained after 2, 4, 7, and 14 days in culture. Scale bars: $200 \mu \mathrm{m}$ (A-D) and $100 \mu \mathrm{m}$ (E-H). (I-K) Numbers of cell clusters greater than $50 \mu \mathrm{m}$ in diameter in neurosphere cultures derived from areas a-f (see Figure 1) on culture day 9. (I) Subventricular zone (SVZ); (J) striatum; (K) cortex. HIE ipsilateral and contralateral areas: $n=6$; sham-operated: $n=6$. Statistical comparisons among groups (1. sham-operated 2. HIE contralateral side 3. HIE ipsilateral side) were performed using the Games-Howell test or Mann-Whitney $U$ test. Data are shown as mean \pm SEM. $\star \star: 1$ vs. 3 , $\star \star$ or $\star: 1$ vs. 2 or 2 vs. 3. (L) Proliferation of cells in the SVZ of sham-operated animals and the contralateral (HIE contra) or ipsilateral (HIE ipsi) SVZ of HIE model animals, as measured by the MTT assay. (I-L) $\star P<0.05$ and $\star \star P<0.01$. (M-P) Proliferating cell in the D4 cell clusters incorporated BrdU and formed secondary neurosphere-like cell clusters. (M) Proliferating cells stained for BrdU (L: green) observed in the D4 cell cluster. (N-P) Secondary neurosphere-like cell cluster formed from a single cell of the D4 cell clusters after 7,14 , and 30 days in culture. Scale bars: $100 \mu \mathrm{m}$.

\section{Results}

Cell cluster formation by cells derived from ischemia-injured areas in HIE model rats

Three days after HIE induction, the ischemia-injured area was observed as a white focal region in the ischemic hemisphere; this area was confirmed as the infarct area using TTC staining (Figures 1B-1D). Equal volumes of liquefied tissues were aspirated from the ischemiainjured area using a 29G needle at 3,5, and 7 days after HIE induction, then extracted and isolated using a $29 \mathrm{G}$ needle for subsequent cell culture using the neurosphere assay. The largest numbers of cell clusters 
from ischemia-injured areas were observed 3 days after HIE induction. The ischemia-injured hemisphere included more nestin- and/or GFAPpositive cells than the contralateral side 3 days after HIE induction (Supplemental Figure). Therefore, we extracted cells from injured tissue 3 days after HIE induction in subsequent experiments.

On cell culture day 2 , some cell clusters were visualized in cultures derived from the ipsilateral (ischemic) cortex (area $c$ in Figure 1A); these clusters showed increases in cell number and cluster diameter over the next 2 days. On culture day 4, some cell clusters showed neurosphere-like morphology (Figures $2 \mathrm{~B}$ and 2F, black arrows). The diameters of neurosphere-like cell clusters increased with the adhesion of each cluster through culture day 14 (Figures 2C, 2D, 2G and 2H). The number of neurosphere-like cell clusters diminished with cluster adhesion (Figures 2B-2D).

\section{Change in the number of cell clusters}

To demonstrate changes in the number of cell clusters, clusters over $50 \mu \mathrm{m}$ in diameter were counted under a microscope on cell culture days $2,4,6,7,8$ and 9. Few cell clusters in cultures derived from the SVZ of sham-operated animals could be detected on culture day 2 , and the number of clusters gradually increased through culture day 7 (127.33 \pm 13.04 clusters/well, $n=12$; Figure 2I, closed triangles). Few cell clusters could be detected in cultures derived from the cerebral cortex and striatum of sham-operated animals on each observation day (Figures 2J and 2K, closed triangles).

Cells derived from the ipsilateral cortex, striatum, and SVZ of HIE model rats formed clusters on culture day 2 (Figures $2 \mathrm{I}-2 \mathrm{~K}$, closed diamonds). The numbers of cell clusters derived from each of these regions dramatically increased and peaked at culture day 4 (SVZ: $279.17 \pm 65.76$ clusters/well; cerebral cortex: $193.33 \pm 32.49$ clusters/ well; striatum: $253.83 \pm 73.74$ clusters/well; $n=6$ for each) and then continuously decreased through culture day 9 (Figures 2I-2K, closed diamonds).

Cell cluster numbers in cultures derived from the contralateral SVZ of the HIE group were very similar to those of the sham-operated group (Figure 2I, closed squares). The small numbers of cell clusters in cultures derived from the contralateral cortex and striatum were also very similar to those of the sham-operated group (Figures $2 \mathrm{~J}$ and $2 \mathrm{~K}$, closed squares).

\section{Increased proliferative capacity of cells derived from the SVZ of HIE model rats}

To determine the reason for the observed increase in cell clusters derived from the ischemic hemisphere of HIE model animals, MTT assays were performed to measure the mitotic activity of cells derived from the SVZs of sham control animals and the ipsilateral and contralateral hemispheres of HIE model animals. Absorbance values were normalized to those of the sham-operated control group. The mean $( \pm$ SE) normalized absorbance values were $1.00 \pm 0.06(n=12)$ for sham-operated animals and $2.56 \pm 0.20(n=6)$ and $1.19 \pm 0.07(n=6)$ for the ipsilateral and contralateral hemispheres, respectively, of HIE model animals. The mitotic activity of cells derived from the ipsilateral hemisphere was significantly greater than that of cells derived from the contralateral hemisphere and sham-operated controls $(P<0.001$ for both comparisons).

\section{Properties of D4 cell clusters derived from infarct cortex}

To confirm whether cell clusters derived from the ischemia-injured cerebral cortex have properties of neural stem cells, we analyzed clusters derived from ischemia-injured areas after 4 days in culture (D4 cell clusters). To investigate the proliferation potential of the clustered cells, we labeled the D4 cell clusters with BrdU and detected BrdU positive cells in the D4 cell clusters (D4 cell clusters, Figure 2M).

Next, to investigate the self-renewal capacity of cells derived from the ischemic cortex, single cells isolated from D4 cell clusters were placed in single wells of a 96-well plate and cultured using the neurosphere culture protocol. These cells divided and formed clusters, the size of which increased with a morphological pattern very similar to that of a neurosphere (Figures $2 \mathrm{~N}-2 \mathrm{P}$ ) [31,32].

We performed immunocytochemical analyses to assess the potential of cells in the D4 clusters to differentiate into neurons, astrocytes, and oligodendrocytes. $\mathrm{D} 4$ cell clusters included cells positive for the neuronal progenitor cell markers nestin and $\beta$-III-tubulin, the astrocyte and astrocyte progenitor cell markers GFAP and vimentin, and the oligodendrocyte progenitor cell markers O4 and NG2 (Figures 3A-3F). Moreover, some cells expressed the activated microglial marker Ibal (Figure 3G). To clarify the genetic identity of cells in the undifferentiated D4 clusters, RT-PCR analyses were performed. D4 cell clusters expressed mRNA for NANOG, Sox2, Oct3/4, and Rex1, but not KLF4 (Figure 3H).

\section{Differentiation of D4 cell clusters derived from the infarct cortex}

Differentiation was induced in D4 cell clusters by omitting EGF and bFGF from the culture media. Immunocytochemistry was performed using antibodies against $\beta$-III-tubulin, MAP2, GFAP, and $\mathrm{O} 4$, which are specific markers for neuronal progenitor cells, mature neurons, astrocytes, and oligodendrocytes, respectively (Figures 3I-3L). Expression of mRNAs for nestin, $\beta$-III-tubulin, GFAP, and MBP in the differentiated $\mathrm{D} 4$ cell clusters was confirmed by RT-PCR (Figure 3M).

\section{Discussion}

In this study, we demonstrate that infarct brain areas in HIE model rats contain cells that can proliferate and form neurosphere-like clusters. In addition, these cells can differentiate into neurons, astrocytes, and oligodendrocytes. To our knowledge, this is the first report showing that endogenous neural stem and progenitor cells can be isolated from ischemia-injured brain areas of HIE animal models.

Neural stem and progenitor cells have been reported in the SVZ of the neonatal brain and are increased by hypoxic-ischemic injury [42]. We demonstrated that cells obtained not only from the ipsilateral SVZ in HIE model animals but also from the infarct cortex and striatum could form cell clusters that increased in diameter over time. Moreover, we observed that different types of cells formed clusters and that clusters fused with each other and enlarged (Figure 2). This phenomenon was similar to the formation of neurospheres [43]. Therefore, we conclude that many cells similar to neural stem and progenitor cells exist in infarct brain areas of HIE model animals.

In cultures derived from infarct areas in HIE model rats, cell clusters greater than $50 \mu \mathrm{m}$ in diameter were dramatically increased after 4 days in culture. This observation suggests that the proliferative capacity of neural stem and progenitor cells in infarct areas is increased by hypoxic-ischemic injury. Neural stem cells propagate and double approximately every $20 \mathrm{~h}$ in neurosphere culture assays [44]. Mori et al. [45] reported that when neural stem and progenitor cells were seeded at a density of $1 \times 10^{4}$ cells/well, the number of neurospheres greater than $30 \mu \mathrm{m}$ in diameter rapidly increased over $72 \mathrm{~h}$ in culture. 
Citation: Kido J, Matsumoto T (2016) Neural Stem and Progenitor Cells with High Proliferation Potential in Injured Areas in a Model of Neonatal Hypoxic-Ischemic Encephalopathy. J Neurol Neurophysiol 7: 348. doi:10.4172/2155-9562.1000348
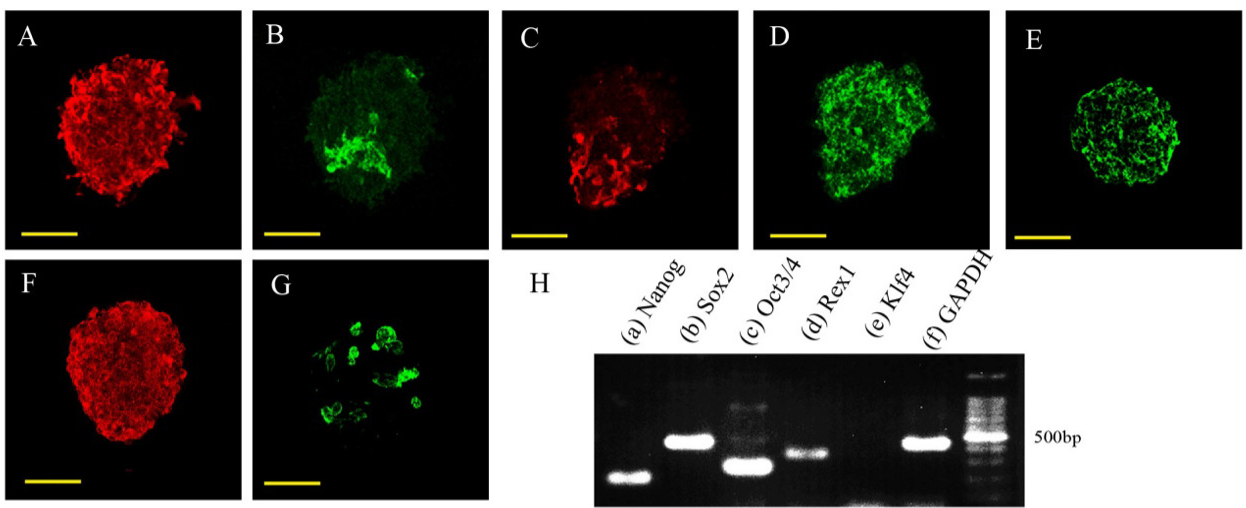

$\mathrm{H}$
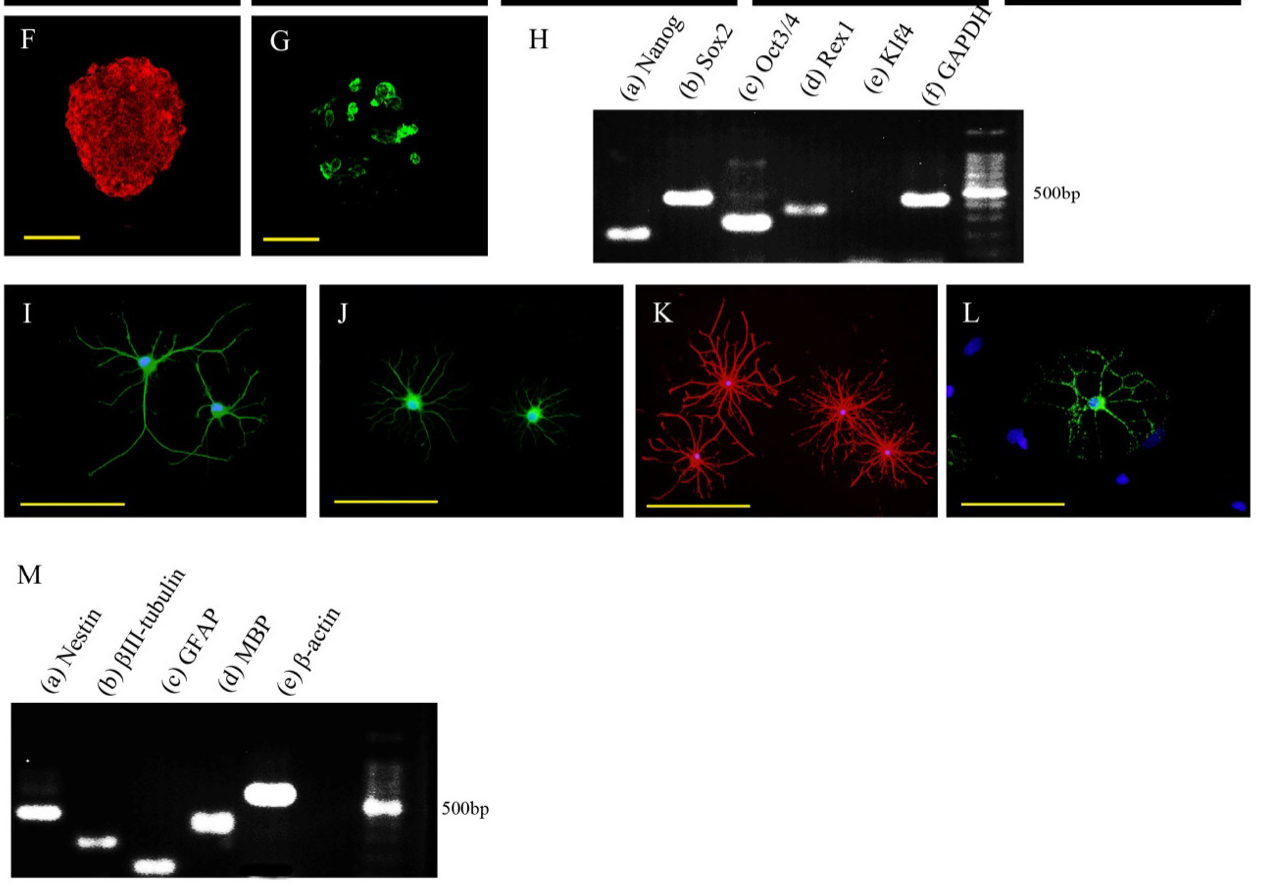

Figure 3: Characterization of D4 cell clusters derived from the infract cortex area and differentiation of D4 cell clusters. (A-G) Immunocytochemistry of D4 cell clusters. (A) nestin; (B) neural/glial antigen 2 (NG2); (C) glial fibrillary acidic protein (GFAP); (D) B-III-tubulin; (E) oligodendrocyte marker O4; (F) vimentin; (G) ionizing calcium-binding adaptor molecule 1 (lba1). Scale bars: $50 \mu \mathrm{m}$. (H) RT-PCR analyses showing expression of (a) NANOG, (b) (sex determining region Y)-box 2 (Sox2), (c) octamer-binding transcription factor 3/4 (Oct3/4), and (d) Rex1 in D4 cell clusters. Expression of KLF4 (e) was not observed. (f) Glyceraldehyde-3-phosphate dehydrogenase (GAPDH) internal control. (I-L) Immunocytochemistry of differentiated cells from D4 cell clusters. (I) $\beta$-III-tubulin (green); (J) microtubule-associated protein 2 (MAP2; green); (K) GFAP (red); and (L) O4 (green). Nuclei were counterstained with 4',6-diamidino-2-phenylindole (DAPI; blue). Scale bars: 50 um (I, J, and L) and $100 \mu \mathrm{m}(\mathrm{K})$. (M) RT-PCR analyses showing expression of (a) nestin, (b) $\beta$-III-tubulin, (c) GFAP, and (d) myelin basic protein (MBP) in differentiated D4 cell clusters. (e) $\beta$-actin internal control.

The neurospheres then increased in diameter but decreased in number. Similar to their results, our study also showed that the number of cell clusters in cultures derived from infarct areas rapidly increased over 4 days in culture.

Proliferation of neural stem and progenitor cells in the SVZ in vivo is stimulated by hypoxic conditions [46]. Here, the MTT assay demonstrated that cells derived from the ipsilateral SVZ of HIE model rats increased at a rate 2.56 times greater than that of cells derived from the SVZ of sham-operated animals. A previous study reported that the induction of hypoxia-inducible transcription factor-1 alpha by hypoxia enhanced the proliferation of neural stem and progenitor cells [47]. Therefore, humoral factors such as hypoxia-inducible transcription factor-1 alpha may contribute to the enhanced proliferation of cells derived from the ipsilateral SVZ of HIE model animals. In addition, Deierborg et al. reported that mitogenic factors secreted from activated microglia, which migrate to injured brain areas, enhanced the proliferation potential of neural stem and progenitor cells [48]. In our neurosphere assay, cells obtained from infarct areas were cultured with activated microglia. This method may contribute to the accelerated formation and proliferation of cell clusters in cultures obtained from HIE model rats.
Similar to cells obtained from infarct brain areas in our HIE model, cells derived from the infarct cortex in a mouse model of adult middle carotid artery dissection form cell clusters in neurosphere assays [25]. Nakagomi et al. reported that the number of cell clusters greater than $80 \mu \mathrm{m}$ in diameter increased over 14 days in culture, and the number of clusters observed on day 14 was 8 times greater than on that on day 2 [25]. We analyzed cell clusters greater than $50 \mu \mathrm{m}$ in diameter; the number of cell clusters increased by 36 times in the ipsilateral SVZ, by 30 times in the infarct striatum, and by 6 times in the infarct cortex from culture day 2 to day 4 . Secondary neurosphere-like cell clusters formed at a rate of 15 wells per 1000 wells in our study, which is 3 times greater than that found by Nakagomi et al. using an adult infarction model. These increases in the formation of primary and secondary cell clusters may be due to differences in species, age, and disease model.

D4 cell clusters expressed markers of 3 neural lineages, as demonstrated by immunocytochemistry, and expressed mRNAs for NANOG, Sox2, Oct3/4, and Rex1. These mRNAs are expressed in embryonic stem cells, and several studies have demonstrated the expression of NANOG and Sox 2 in neural stem cells; however, expression patterns of Oct $3 / 4$ and Rex 1 varied in these reports $[44,49,50]$. Suslov et al. [51] reported that differentiation states and 
Citation: Kido J, Matsumoto T (2016) Neural Stem and Progenitor Cells with High Proliferation Potential in Injured Areas in a Model of Neonatal Hypoxic-Ischemic Encephalopathy. J Neurol Neurophysiol 7: 348. doi:10.4172/2155-9562.1000348

gene expression patterns of neurosphere cells differed depending on the type and size of the neurosphere and the position of the cell within the neurosphere.

In conclusion, we demonstrate the presence of numerous neural stem and progenitor cells with enhanced proliferative potential in ischemia-injured brain areas of a rat model of HIE. Activation of neural stem and progenitor cells in infarct brain areas may contribute to neuroprotection and regeneration. Therefore, neural stem and progenitor cells may represent an important treatment option for HIE.

\section{Acknowledgement}

This study was supported in part by a Grant-in-Aid from the Global COE Program of the Japanese Society for the Promotion of Science and the Ministry of Education, Culture, Sports, Science and Technology; a Grant-in-Aid for Pediatric Research from the Ministry of Health, Labour and Welfare; and a Grant-in-Aid for Scientific Research from the Ministry of Education, Culture, Sports, Science and Technology. We are grateful to Drs. Masanori Iwai and Shirou Matsumoto for their help and advice regarding this study.

\section{References}

1. Kurinczuk JJ, White-Koning M, Badawi N (2010) Epidemiology of neonatal encephalopathy and hypoxic-ischaemic encephalopathy. Early Hum Dev 86: 329-338

2. Edwards AD, Brocklehurst $P$, Gunn AJ, Halliday H, Juszczak E, et al. (2010) Neurological outcomes at 18 months of age after moderate hypothermia for perinatal hypoxic ischaemic encephalopathy: synthesis and meta-analysis of trial data. BMJ 340: c363.

3. Tagin MA, Woolcott CG, Vincer MJ, Whyte RK, Stinson DA (2012) Hypothermia for neonatal hypoxic ischemic encephalopathy: an updated systematic review and meta-analysis. Arch Pediatr Adolesc Med 166: 558-566.

4. Kracer B, Hintz SR, Van Meurs KP, Lee HC (2014) Hypothermia therapy for neonatal hypoxic ischemic encephalopathy in the state of California. J Pediatr 165: $267-273$.

5. Doetsch F, García-Verdugo JM, Alvarez-Buylla A (1997) Cellular composition and three-dimensional organization of the subventricular germinal zone in the adult mammalian brain. J Neurosci 17: 5046-5061.

6. Alvarez-Buylla A, Garcia-Verdugo JM (2002) Neurogenesis in adult subventricular zone. J Neurosci 22: 629-634.

7. Daval JL, Pourié G, Grojean S, Lièvre V, Strazielle C, et al. (2004) Neonatal hypoxia triggers transient apoptosis followed by neurogenesis in the rat CA1 hippocampus. Pediatr Res 55: 561-567.

8. Liu J, Solway K, Messing RO, Sharp FR (1998) Increased neurogenesis in the dentate gyrus after transient global ischemia in gerbils. J Neurosci 18: 7768 7778 .

9. Jin K, Minami M, Lan JQ, Mao XO, Batteur S, et al. (2001) Neurogenesis in dentate subgranular zone and rostral subventricular zone after focal cerebral ischemia in the rat. Proc Natl Acad Sci U S A 98: 4710-4715.

10. Nakatomi H, Kuriu T, Okabe S, Yamamoto S, Hatano O, et al. (2002) Regeneration of hippocampal pyramidal neurons after ischemic brain injury by recruitment of endogenous neural progenitors. Cell 110: 429-441.

11. Jin K, Sun Y, Xie L, Peel A, Mao XO, et al. (2003) Directed migration of neuronal precursors into the ischemic cerebral cortex and striatum. Mol Cell Neurosci 24: 171-189.

12. Hayashi T, Iwai M, Ikeda T, Jin G, Deguchi K, et al. (2005) Neural precursor cells division and migration in neonatal rat brain after ischemic/hypoxic injury. Brain Res 1038: 41-49.

13. Iwai M, Sato K, Omori N, Nagano I, Manabe Y, et al. (2002) Three steps of neural stem cells development in gerbil dentate gyrus after transient ischemia. J Cereb Blood Flow Metab 22: 411-419.

14. Iwai M, Sato K, Kamada H, Omori N, Nagano I, et al. (2003) Temporal profile of stem cell division, migration, and differentiation from subventricular zone to olfactory bulb after transient forebrain ischemia in gerbils. J Cereb Blood Flow Metab 23: 331-341.

15. Arvidsson A, Collin T, Kirik D, Kokaia Z, Lindvall O (2002) Neuronal replacement from endogenous precursors in the adult brain after stroke. Nat Med 8: 963-970.
16. Ikeda T, Iwai M, Hayashi T, Nagano I, Shogi M, et al. (2005) Limited differentiation to neurons and astroglia from neural stem cells in the cortex and striatum after ischemia/hypoxia in the neonatal rat brain. Am J Obstet Gynecol 193: 849-856.

17. Plane JM, Liu R, Wang TW, Silverstein FS, Parent JM (2004) Neonatal hypoxicischemic injury increases forebrain subventricular zone neurogenesis in the mouse. Neurobiol Dis 16: 585-595.

18. Iwai M, Cao G, Yin W, Stetler RA, Liu J, et al. (2007) Erythropoietin promotes neuronal replacement through revascularization and neurogenesis after neonatal hypoxia/ischemia in rats. Stroke 38: 2795-2803.

19. Rogers EE, Bonifacio SL, Glass HC, Juul SE, Chang T, et al. (2014) Erythropoietin and hypothermia for hypoxic-ischemic encephalopathy. Pediatr Neurol 51: 657-662.

20. Shintani S, Murohara T, Ikeda H, Ueno T, Honma T, et al. (2001) Mobilization of endothelial progenitor cells in patients with acute myocardial infarction. Circulation 103: 2776-2779.

21. Oliver JA, Maarouf O, Cheema FH, Martens TP, Al-Awqati Q (2004) The renal papilla is a niche for adult kidney stem cells. J Clin Invest 114: 795-804.

22. Humphreys BD, Valerius MT, Kobayashi A, Mugford JW, Soeung S, et al. (2008) Intrinsic epithelial cells repair the kidney after injury. Cell Stem Cell 2: 284-291.

23. Suga $\mathrm{H}$, Eto $\mathrm{H}$, Aoi $\mathrm{N}$, Kato $\mathrm{H}$, Araki J, et al. (2010) Adipose tissue remodeling under ischemia: death of adipocytes and activation of stem/progenitor cells. Plast Reconstr Surg 126: 1911-1923.

24. Rumman M, Dhawan J, Kassem M, et al. (2015) Concise Review: Quiescence in Adult Stem Cells: Biological Significance and Relevance to Tissue Regeneration. Stem Cells 33: 2903-2912.

25. Nakagomi T, Taguchi A, Fujimori Y, Saino O, Nakano-Doi A, et al. (2009) Isolation and characterization of neural stem/progenitor cells from post-stroke cerebral cortex in mice. Eur J Neurosci 29: 1842-1852.

26. Landis SC, Amara SG, Asadullah K, Austin CP, Blumenstein R, et al. (2012) A call for transparent reporting to optimize the predictive value of preclinical research. Nature 490: 187-191.

27. Lapchak PA (2013) Recommendations and practices to optimize stroke therapy: developing effective translational research programs. Stroke 44: 841-843.

28. Levine S (1960) Anoxic-ischemic encephalopathy in rats. Am J Pathol 36: 1-17.

29. Vannucci RC, Vannucci SJ (2005) Perinatal hypoxic-ischemic brain damage: evolution of an animal model. Dev Neurosci 27: 81-86.

30. Joshi CN, Jain SK, Murthy PS (2004) An optimized triphenyltetrazolium chloride method for identification of cerebral infarcts. Brain Res Brain Res Protoc 13 11-17.

31. Reynolds BA, Weiss S (1992) Generation of neurons and astrocytes from isolated cells of the adult mammalian central nervous system. Science 255 : 1707-1710.

32. Reynolds BA, Weiss S (1996) Clonal and population analyses demonstrate that an EGF-responsive mammalian embryonic CNS precursor is a stem cell. Dev Biol 175: 1-13.

33. Santa-Olalla J, Covarrubias L (1999) Basic fibroblast growth factor promotes epidermal growth factor responsiveness and survival of mesencephalic neural precursor cells. J Neurobiol 40: 14-27.

34. Kanemura Y, Mori H, Kobayashi S, Islam O, Kodama E, et al. (2002) Evaluation of in vitro proliferative activity of human fetal neural stem/progenitor cells using indirect measurements of viable cells based on cellular metabolic activity. $J$ Neurosci Res 69: 869-879.

35. Tervonen TA, Ajamian F, De Wit J, Verhaagen J, Castrén E, et al. (2006) Overexpression of a truncated TrkB isoform increases the proliferation of neural progenitors. Eur J Neurosci 24: 1277-1285.

36. Li Z, Zhang Y, Li D, Feng Y (2000) Destabilization and mislocalization of myelin basic protein mRNAs in quaking dysmyelination lacking the QKI RNA-binding proteins. J Neurosci 20: 4944-4953.

37. Suzuki H, Taguchi T, Tanaka H, Kataoka H, Li Z, et al. (2004) Neurospheres induced from bone marrow stromal cells are multipotent for differentiation into neuron, astrocyte, and oligodendrocyte phenotypes. Biochem Biophys Res Commun 322: 918-922.

38. Lu J, Moochhala S, Moore XL, Ng KC, Tan MH, et al. (2006) Adult bone marrow cells differentiate into neural phenotypes and improve functional recovery in rats following traumatic brain injury. Neurosci Lett 398: 12-17. 
Citation: Kido J, Matsumoto T (2016) Neural Stem and Progenitor Cells with High Proliferation Potential in Injured Areas in a Model of Neonatal Hypoxic-Ischemic Encephalopathy. J Neurol Neurophysiol 7: 348. doi:10.4172/2155-9562.1000348

39. Li W, Wei W, Zhu S, Zhu J, Shi Y, et al. (2009) Generation of rat and human induced pluripotent stem cells by combining genetic reprogramming and chemical inhibitors. Cell Stem Cell 4: 16-19.

40. Kawamata M, Ochiya T (2010) Generation of genetically modified rats from embryonic stem cells. Proc Natl Acad Sci U S A 107: 14223-14228.

41. Song N, Jia XS, Jia LL, Ma XB, Li F, et al. (2010) Expression and role of Oct3/4, Nanog and Sox2 in regeneration of rat tracheal epithelium. Cell Prolif 43: 49-55.

42. Iwai M, Ikeda T, Hayashi T, Sato K, Nagata T, et al. (2006) Temporal profile of neural stem cell proliferation in the subventricular zone after ischemia/hypoxia in the neonatal rat brain. Neurol Res 28: 461-468.

43. Mori H, Fujitani T, Kanemura Y, Kino-Oka M, Taya M (2007) Observational examination of aggregation and migration during early phase of neurosphere culture of mouse neural stem cells. J Biosci Bioeng 104: 231-234.

44. Ahmed S (2009) The culture of neural stem cells. J Cell Biochem 106: 1-6.

45. Mori H, Ninomiya K, Kino-oka M, Shofuda T, Islam MO, et al. (2006) Effect of neurosphere size on the growth rate of human neural stem/progenitor cells. $J$ Neurosci Res 84: 1682-1691.
46. Yang Z, Levison SW (2006) Hypoxia/ischemia expands the regenerative capacity of progenitors in the perinatal subventricular zone. Neuroscience 139 : 555-564.

47. Zhao T, Zhang CP, Liu ZH, Wu LY, Huang X, et al. (2008) Hypoxia-driven proliferation of embryonic neural stem/progenitor cells--role of hypoxiainducible transcription factor-1alpha. FEBS J 275: 1824-1834.

48. Deierborg T, Roybon L, Inacio AR, Pesic J, Brundin P (2010) Brain injury activates microglia that induce neural stem cell proliferation ex vivo and promote differentiation of neurosphere-derived cells into neurons and oligodendrocytes. Neuroscience 171: 1386-1396.

49. Lengner CJ, Camargo FD, Hochedlinger K, Welstead GG, Zaidi S, et al. (2007) Oct4 expression is not required for mouse somatic stem cell self-renewal. Cell Stem Cell 1: 403-415.

50. Conti L, Cattaneo E (2010) Neural stem cell systems: physiological players or in vitro entities? Nat Rev Neurosci 11: 176-187.

51. Suslov ON, Kukekov VG, Ignatova TN, Steindler DA (2002) Neural stem cell heterogeneity demonstrated by molecular phenotyping of clonal neurospheres. Proc Natl Acad Sci U S A 99: 14506-14511. 